\title{
Snat: a SNP annotation tool for bovine by integrating various sources of genomic information
}

Jicai Jiang, Li Jiang, Bin Zhou, Weixuan Fu, Jian-Feng Liu* and Qin Zhang*

\begin{abstract}
Background: Most recently, with maturing of bovine genome sequencing and high throughput SNP genotyping technologies, a large number of significant SNPs associated with economic important traits can be identified by genome-wide association studies (GWAS). To further determine true association findings in GWAS, the common strategy is to sift out most promising SNPs for follow-up replication studies. Hence it is crucial to explore the functional significance of the candidate SNPs in order to screen and select the potential functional ones. To systematically prioritize these statistically significant SNPs and facilitate follow-up replication studies, we developed a bovine SNP annotation tool (Snat) based on a web interface.

Results: With Snat, various sources of genomic information are integrated and retrieved from several leading online databases, including SNP information from dbSNP, gene information from Entrez Gene, protein features from UniProt, linkage information from AnimalQTLdb, conserved elements from UCSC Genome Browser Database and gene functions from Gene Ontology (GO), KEGG PATHWAY and Online Mendelian Inheritance in Animals (OMIA). Snat provides two different applications, including a CGI-based web utility and a command-line version, to access the integrated database, target any single nucleotide loci of interest and perform multi-level functional annotations. For further validation of the practical significance of our study, SNPs involved in two commercial bovine SNP chips, i.e., the Affymetrix Bovine 10K chip array and the Illumina 50K chip array, have been annotated by Snat, and the corresponding outputs can be directly downloaded from Snat website. Furthermore, a real dataset involving 20 identified SNPs associated with milk yield in our recent GWAS was employed to demonstrate the practical significance of Snat.

Conclusions: To our best knowledge, Snat is one of first tools focusing on SNP annotation for livestock. Snat confers researchers with a convenient and powerful platform to aid functional analyses and accurate evaluation on genes/variants related to SNPS, and facilitates follow-up replication studies in the post-GWAS era.
\end{abstract}

\section{Background}

Currently, genome wide association studies (GWAS) have been widely accepted as a primary approach for gene identification concerning complex traits. A subset of SNPs related to the trait of interest can be derived from GWAS at a specified level of statistical significance. To further determine true association findings in GWAS, the common strategy is to sift out the most promising SNPs for follow-up replication studies. Hence

\footnotetext{
*Correspondence: liujf@cau.edu.cn; qzhang@cau.edu.cn Key Laboratory of Animal Genetics and Breeding of the Ministry of Agriculture, College of Animal Science and Technology, China Agricultural University, Beijing, 100193, P.R. China
}

it is crucial to explore the functional significance of the candidate SNPs in order to screen and select the potential functional ones. So far, a variety of public bioinformatics databases, e.g., NCBI Entrez Gene [1], UniProt [2], Gene Ontology [3], KEGG PATHWAY [4] and AnimalQTLdb [5], etc., contain different aspects of biological information required for SNP functional annotation. However, it is infeasible to mine the relevant research data from these public sources by a single query. Furthermore, it is a daunting task to integrate various sources of functional information of interest among the large assortment of data in a manual fashion. 
To deal with SNP annotation, a number of bioinformatics tools have been created. However, the majority of these tools are for humans, e.g., SNPit [6], SNPnexus [7], and few tools are available for other species such as bovine. FunctSNP [8] is currently the only tool available for bovine SNP annotation. Although FunctSNP provides various functions to search and manage annotated data related to SNPs, the main limitations maybe exist: Firstly, it is not straightforward and convenient to annotate even one SNP using FunctSNP, because users should initially construct a local database before SNP annotation and cannot be run online through a web interface, leading to low efficiency and time consuming. Secondly, FunctSNP merely outputs some identifiers relating to some public databases in queries, e.g. GO:0000122 (GO term), bta00340 (KEGG Pathway entry), A7YWP4 (UniProt accession), and more detailed descriptions for these identifiers should be obtained manually via accessing the relevant public domains. Finally, FunctSNP only recognizes the cluster ID (rs\#) and accurate position of a SNP in dbSNP, triggering an obviously application limitation since users are usually interested in those SNPs without specific cluster IDs or not included in dbSNP in most cases, such that FunctSNP can not deal with these SNPs under this situation.

Focusing on tackling the above limitations, we developed a SNP annotation tool (Snat) to provide a wide array of functional SNP annotations for bovine. In Snat, the most novelty is that recent versions of information from FTPs and webpages of the public domains have been extracted and integrated to construct a composite database. Furthermore, SNPs at arbitrary positions can be well annotated through a single query, regardless of whether they are involved in the dbSNP database or not.

\section{Implementation}

Snat aims at the design for a novel query scheme which can provide precise and comprehensive annotation for bovine SNPs. This would be helpful for mining potential clues of functional importance. Snat is written in Perl as well as SQL scripts with modular architecture. The design of the query scheme with common interfaces supports multiple options for each annotation task.

Developing of Snat consists of two major steps: The first step is the construction of the local database that contains substantial information for SNP annotation. Specifically, documents are retrieved from various aspects of online databases using an automated procedure via Perl program. These online resources include dbSNP [9], Entrez Gene, UniProt, GO, KEGG PATHWAY, AnimalQTLdb, UCSC ConsElements [10,11] and OMIA [12]. In order to ensure the accuracy of the online information, Snat integrates packaged data downloaded from FTPs as well as data from webpages (see Figure 1 and Figure 2 for detailed architecture). Subsequently, a local MySQL relational database can be constructed from the retrieved information via SQL and Perl scripts. The second step is the design of a user friendly client-side which can implement SNP annotation with multiple choices. Programs are written in Perl. Users can browse website of Snat to perform online SNP annotation. The annotation results can be viewed in colored and aligned HTML tables, as well as printed in a plain text and downloaded as a text or compressed file.

Besides web-based application of Snat, we also developed an alternative version of Snat in command-line mode, which can be run locally to query and annotate SNPs. Specifically, via downloading the integrated database from the Snat site as a SQL script, users can construct the local database, which is identical with that of web-based version. Based on the local database, SNP annotation can be conducted to print results into a text file via Perl scripts in a command-line fashion. The raw text report, generated by either the locally running Perl programs or downloaded from Snat website, looks not well-formatted but is created with a specified access rule. Thus it can be readily processed via running a Perl program provided by Snat website to produce annotation data structured in TSV files for further analyses.

\section{Results}

Features

Snat addresses two functions. One is to query and annotate those SNPs involved in dbSNP, the other is to deal with arbitrary single nucleotide loci no matter whether they are included in dbSNP or not (see Additional file 1 ). The interface of Snat for web services consists of three parts: input, annotation options and output options (Figure 3). Users can input either coordinate or rs-identifier of individual SNP, or upload an index file containing a bunch of SNPs in a batch fashion, to perform annotation. By setting specified query options, Snat generates corresponding annotated data with combination of various assortments of biological information of SNPs. The outputs of annotation can be browsed online in HTML or integrated in a plain text file for downloading. Alternatively, Snat provides users with Perl programs running in command-line mode to finish annotation locally. Several options can be set for command-line application. The raw text generated by the locally running annotation programs is identical with that downloaded from online annotation. Compared with outputs in HTML, those stored in the raw text are not well-structured. However, the raw text report is created with a specified format which is readily further processed with programming. A corresponding Perl 


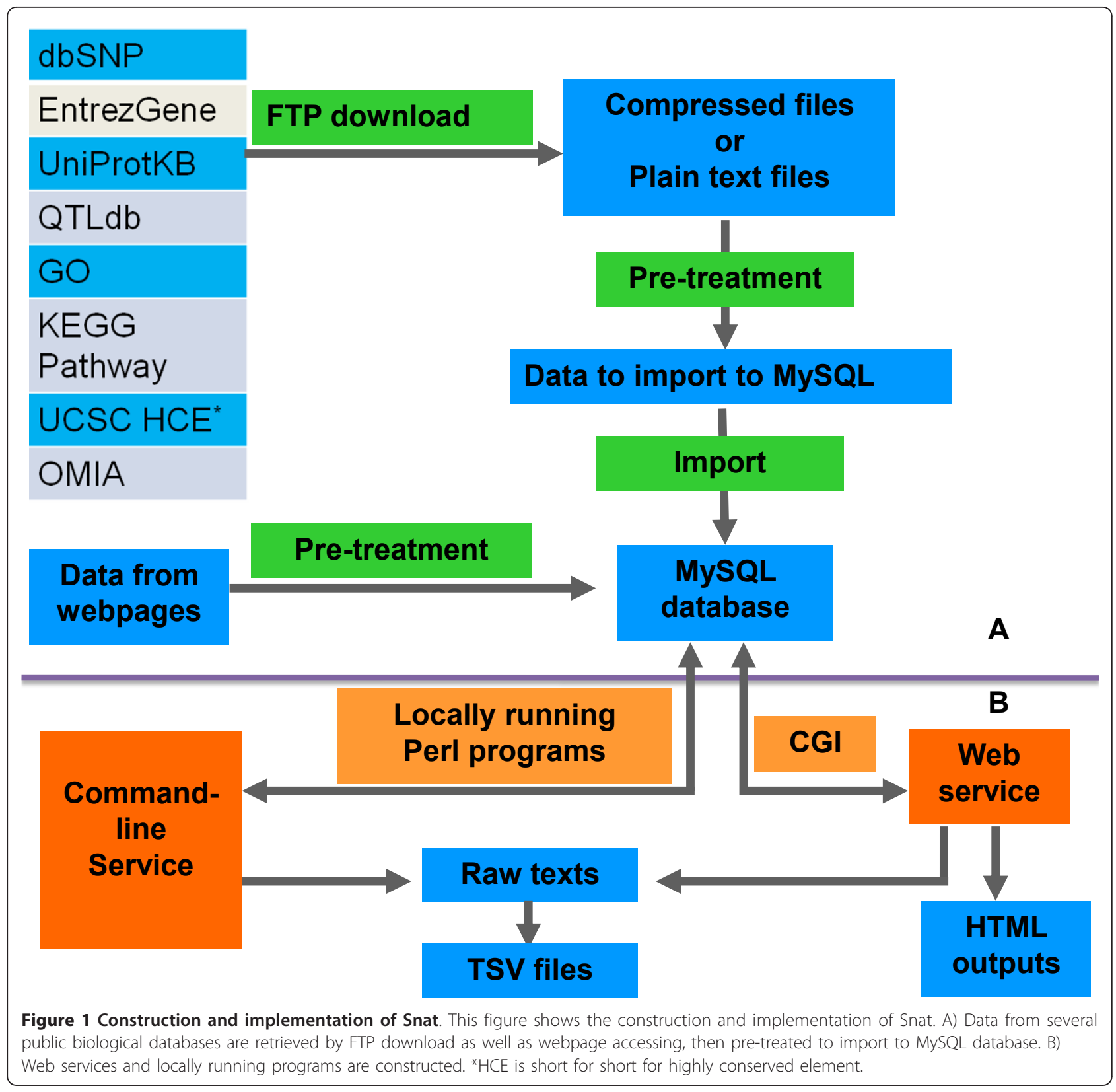

program has been developed to transform the original text to well-formatted data in TSV files that can be clearly viewed by Microsoft Excel and other spreadsheet programs.

Due to incorporating various sources of existing public data into a retrieved database, it is feasible and efficient to obtain annotation data automatically via merely one query in Snat, with seldom need of further re-querying the original public databases manually, facilitating users to rapidly pinpoint these SNPs with functional significance. For further demonstrating the features of Snat, systematic comparisons between Snat and a published tool FunctSNP have been conducted in the following aspects.
First, FunctSNP can only recognize the cluster ID (rs\#) or accurate coordinate of a SNP in dbSNP while Snat can deal with an ambiguous coordinate via searching for the nearest SNP or SNPs within a specified distance away from it (see the first example in Additional file 2).

Second, FunctSNP can only run locally and users should construct a local database before SNP annotation, while Snat provides a locally running commandline version as well as online annotation services. Furthermore, FunctSNP outputs annotation information step by step while Snat produces all output data in a single query (see the second example in Additional file 2). 


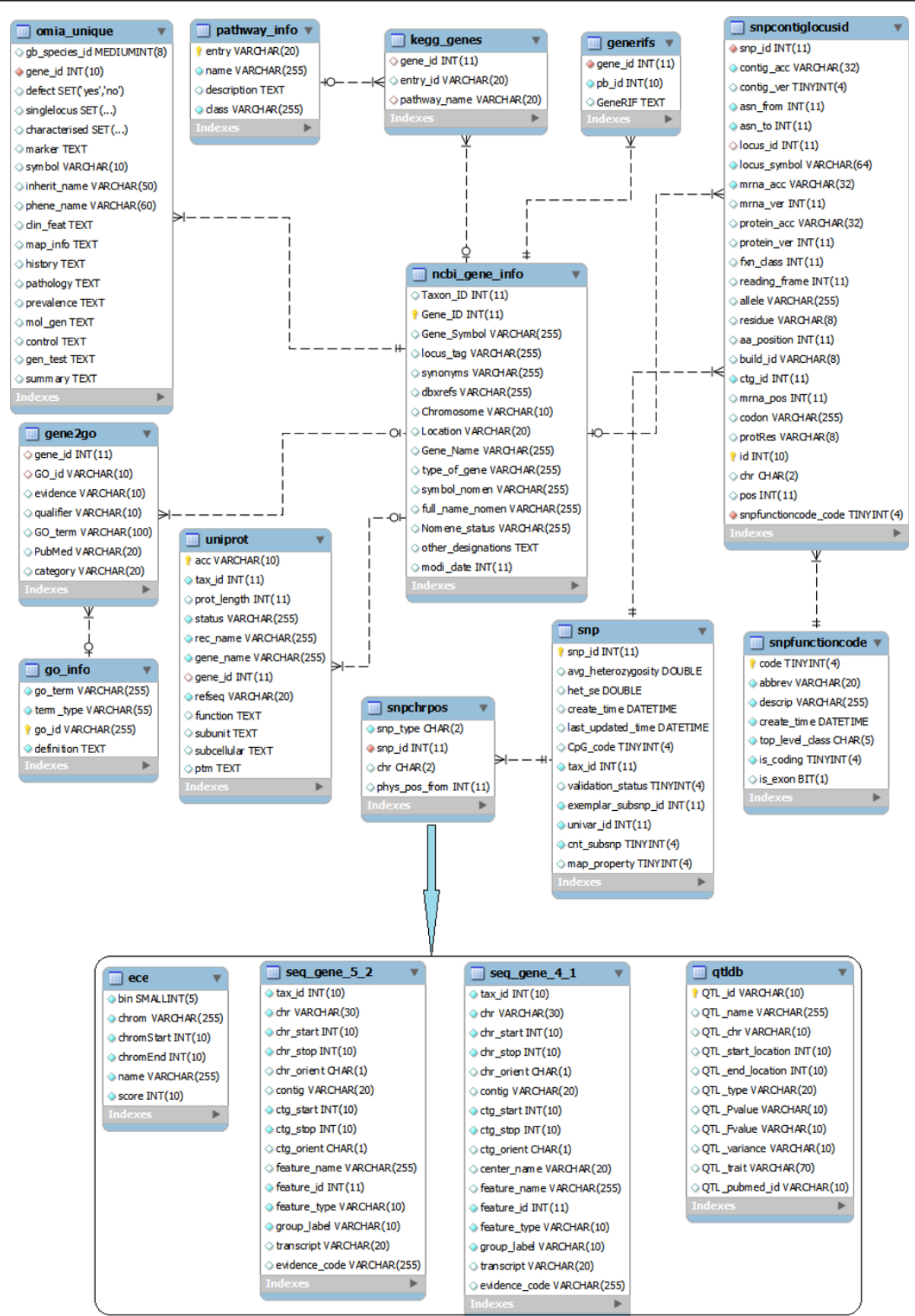

Figure 2 The schema of the database of Snat. The schema shows that all the tables and relationships among them in Snat database.

Third, compared with FunctSNP, Snat integrates extra information of GeneRIFs and conserved elements. Additionally, Snat integrates more detailed information for terms of UniProt Protein, GO, KEGG Pathway and QTL than FunctSNP. Specifically, FunctSNP merely outputs the protein accession and name from UniProt while Snat can output its accession, name, length, function, post-translational modification, etc.

\section{Command-line mode}

Online annotation works well for submitting dozens or hundreds of SNPs in a single query. However, when users need to annotate thousands of SNPs, it is more efficient to use the locally running command-line mode of Snat. Via downloading the integrated database from the Snat site as a SQL script, users can construct the local annotation database. Two Perl programs, named dbSNP.pl and anylocus.pl given by Snat website, can achieve the function of 
Input ( Choose one of the two following approaches )

\begin{tabular}{|l|l|}
\hline Any Locus (Based on Btau4.0) & Chromosome or Scaffold : \\
\hline OR & Position (bp) : \\
\hline Batch Annotation & Please upload a file: (Format examples) \\
\hline
\end{tabular}

Annotation ( Choose any items below to integrate corresponding annotated data )

\begin{tabular}{|c|c|}
\hline NCBI Gene & $\square$ Gene Id $\square$ Symbol $\square$ Name $\square$ Location ( e.g. 18q11) $\square$ Type $\square$ GeneRIFs \\
\hline UniProt Protein & $\begin{array}{l}\square \text { Accession } \square \text { Recommended name } \square \text { Length (AA) } \square \text { Status ( Reviewed or Unreviewed ) } \\
\square \text { Function } \square \text { Subunit structure } \square \text { Subcellular location } \square \text { Post-translational modification }\end{array}$ \\
\hline QTLdb QTL & $\begin{array}{l}\square \text { QTL Id } \square \text { Trait } \square \text { Region (Chr,Start,Stop) } \square \text { Type (e.g. Significant ) } \\
\square \text { P-value } \square \text { F-value } \square \text { Variance } \square \text { PubMed Id (Reference) }\end{array}$ \\
\hline Other infomation & $\begin{array}{l}\square \text { Gene Ontology } \square \text { KEGG pathway } \square \text { Highly conserved elements from UCSC Genome Browser } \\
\square \text { Online Mendelian Inheritance in Animals }\end{array}$ \\
\hline
\end{tabular}

\section{Output}

Output file :

Type of output file : (leave blank to keep output in browser)

- plain text $\bigcirc$ compressed

Figure 3 Web interface of Snat. The web interface of Snat contains three parts: input, annotation and output.

local running. The programs dbSNP.pl and anylocus.pl play the same roles with the modules of "Search SNPs" and "Any Locus" on the webpage, respectively. For demonstrating the application features of local running of Snat, three examples based on command-line mode for performing annotation are given below:

\$ dbSNP.pl -db_name db_name -db_user db_user -db_password db_pw -rs_id rs109234250-all ex1.raw

$\$$ anylocus.pl -db_name name -db_user user -db_password $p w$-chr_pos 14440000 -gene symbol name -uniprot acc function -go outputfile

\$ dbSNP.pl -db_name name -db_user user -db_password $p w$-input inputfile-gene gene_id symbol name generifs -go -kegg -omia -option 30k outputfile

The command line options db_name and db_user, db_password denote the name, the user name and the password of the local annotation database respectively. Input data can be rs-identifier (for example, -rs_id rs 109234250 in the first command), SNP coordinate (for example, -chr_pos 14440000 in the second command) or an index file that contains numbers of SNP coordinates (for example, -input inputfile in the third command). Output reports are generated corresponding to the annotation options in the command. For examples, the option -all means that all annotation information should be included in the output report (see the first command); "-gene symbol name -uniprot acc function -go" means that the symbol and name of gene, the accession and function of UniProt protein and GO information should be included in the output report (see the second command).

\section{Output}

Snat provides output reports in two different formats, i.e., the HTML webpage and the plain text. The HTML output can be clearly viewed online in colored and aligned tables (see Figure 4) while the plain text report is created with a specified format (see Additional file 3). For enhancing readability of annotation data in raw plain text, a Perl program named raw2TSV.pl has been developed to transform the original plain text into several subdata structured in TSV format. An example on how to further process the raw plain text has been given in the following Application subsection. 


\begin{tabular}{|c|c|c|c|c|c|c|c|c|c|c|c|c|}
\hline \multicolumn{13}{|c|}{ >SNP Identifier: rs109234250 } \\
\hline \multicolumn{13}{|c|}{ [SNP information] } \\
\hline \multicolumn{13}{|c|}{ Position: $\mathrm{Chr} 14,445086$, Heterozygosity: 0} \\
\hline GeneID & Function & \multicolumn{2}{|c|}{ Position_in_codon } & Allele & \multicolumn{2}{|c|}{ Protein_residue } & \multicolumn{2}{|c|}{ AA_position } & \multicolumn{4}{|c|}{ Codon_position_on_mRNA } \\
\hline 282609 & missense & \multicolumn{2}{|l|}{1} & A & \multicolumn{2}{|l|}{$\mathrm{T}$} & \multicolumn{2}{|l|}{231} & \multicolumn{4}{|l|}{706} \\
\hline 282609 & cds-reference & \multicolumn{2}{|l|}{1} & $\mathrm{G}$ & \multicolumn{2}{|l|}{ A } & \multicolumn{2}{|l|}{231} & \multicolumn{4}{|l|}{706} \\
\hline \multicolumn{13}{|c|}{ [Gene information] } \\
\hline Gene_Id & \multicolumn{2}{|c|}{ Gene_Symbol } & \multicolumn{2}{|c|}{ Gene_Name } & & & & & \multicolumn{2}{|c|}{ Location } & Type_of_ger & \\
\hline 282609 & DGAT1 & & diacylglyce & cerol O-acyltra & ansferase & & & & - & & protein-codi & \\
\hline [GeneRIFs] & & & & & & & & & & & & \\
\hline PubMed_Id & Description & & & & & & & & & & & \\
\hline 14983021 & $\begin{array}{l}\text { K allele, causing } \\
\text { allele. }\end{array}$ & $g$ an inc & crease in milk fat perc & rcentage in the & live anime & al, is cha & aracterized by a & higher $\mathrm{Vn}$ & $\max$ in $\mathrm{pr}$ & roducing & triglycerides & than the A \\
\hline 15190946 & examined and $\mathrm{ch}$ & haracte & erized the occurrance & e of the K 232 & A substitu & tion in $t$ & the DGAT1 ger & e in Bos $t$ & taurus an & ad Bos in & dicus & \\
\hline 15342525 & Variation in the $\mathrm{n}$ & number & of tandem repeats of & of the $18 \mathrm{mer}$ & element $m$ & ight be & causal for the $v$ & ariability ir & in the tran & nscription & n level of the & DGAT1 gene. \\
\hline [Uniprot Pro & rotein informatio & & & & & & & & & & & \\
\hline Accession $\begin{array}{l}\mathrm{Re} \\
\text { na }\end{array}$ & $\begin{array}{l}\text { Recommended } \\
\text { ame }\end{array}$ & Length & h) Status & Function & & & & & $\begin{array}{l}\text { Subunit } \\
\text { structure }\end{array}$ & Subcell & lular location & \begin{tabular}{|l} 
Post- \\
translational \\
modification
\end{tabular} \\
\hline Q8MK44 & $\begin{array}{l}\text { Diacylglycerol O- } \\
\text { acyltransferase } 1\end{array}$ & 489 & \begin{tabular}{|l} 
Reviewed \\
(UniProtKB/Swiss- \\
Prot)
\end{tabular} & \begin{tabular}{|l|l} 
Catalyzes & CC triacy \\
diacylglyc \\
substrates \\
essential f \\
VLDL (v \\
assembly
\end{tabular} & $\begin{array}{l}\text { s the termi } \\
\text { ylglycerol } \\
\text { cerol and } \\
\text { s. In contr } \\
\text { for CC su } \\
\text { ery low d } \\
\text { (By simil }\end{array}$ & $\begin{array}{l}\text { inal and } \\
\text { synthesi } \\
\text { fatty acy } \\
\text { rast to D } \\
\text { rrvival. } \\
\text { lensity liy } \\
\text { arity). }\end{array}$ & $\begin{array}{l}\text { only committe } \\
\text { is by using } \\
\text { yl CC CoA as } \\
\text { GAT2 it is not } \\
\text { May be involve } \\
\text { poprotein) CC }\end{array}$ & in step in & NA & $\begin{array}{l}\text { Endopl } \\
\text { reticulur } \\
\text { membra } \\
\text { pass CC } \\
\text { protein. }\end{array}$ & $\begin{array}{l}\text { lasmic } \\
\text { an } \\
\text { ane; Multi- } \\
\text { C membrane }\end{array}$ & NA \\
\hline [QTL inform & mation] & & & & & & & & & & & \\
\hline \begin{tabular}{|l|l|} 
QTL Id & Tre \\
\end{tabular} & rait & & & Ty & pe & P-valu & F-value & Variance & Publ & Med_Id & QTL Reg & \\
\hline 6214 & ge at puberty $(\mathrm{EB}$ & & & & gnificant & $<0.05$ & - & - & 1865 & 50300 & Chr14:0- & 5235875 \\
\hline 1731 & 3ody weight (mean & & & & gnificant & - & 12.1 & - & 1553 & 37759 & Chr14:0-2 & 24666782 \\
\hline 10954 & ody weight (wean & ning) & & & gnificant & 2.29 & - & - & ISUO & 0041 & Chr14:0- & 5235875 \\
\hline [GO informa & nation] & & & & & & & & & & & \\
\hline Accession & Term & & Ontology & Definition & & & & & & & & \\
\hline GO:0004144 & $44 \begin{array}{l}\text { diacylglycerol C } \\
\text { acyltransferase } \\
\text { activity }\end{array}$ & & molecular_function C & Catalysis of th & he reactio & $\mathrm{n}$ acyl- $\mathrm{C}$ & $\mathrm{CoA}+1,2-\mathrm{dia}$ & cylglycerol & $\mathrm{ol}=\mathrm{CoA}$ & + triacyl & lglycerol. & \\
\hline GO:0005783 & $33 \begin{array}{l}\text { endoplasmic } \\
\text { reticulum }\end{array}$ & & cellular_component & $\begin{array}{l}\text { The irregular } \\
\text { cytoplasm of } \\
\text { which are ofte } \\
\text { granular), witt }\end{array}$ & $\begin{array}{l}\text { network } \\
\text { many euk } \\
\text { en expand } \\
\mathrm{h} \text { ribosom }\end{array}$ & $\begin{array}{l}\text { of unit m } \\
\text { caryotic } \\
\text { led into } \\
\text { les adhe }\end{array}$ & $\begin{array}{l}\text { embranes, visit } \\
\text { cells. The mem } \\
\text { slitike cavities } \\
\text { ering to the oute }\end{array}$ & $\begin{array}{l}\text { le only by } \\
\text { branes fon } \\
\text { called cist } \\
\text { r surface, }\end{array}$ & $\begin{array}{l}\text { y electron } \\
\text { orm a com } \\
\text { ternae. Th } \\
\text {, and smo }\end{array}$ & $\begin{array}{l}\text { n microsc } \\
\text { aplex mes } \\
\text { he ER tal } \\
\text { oth (with }\end{array}$ & $\begin{array}{l}\text { copy, that oc } \\
\text { shwork of tul } \\
\text { akes two form } \\
\text { h no ribosom }\end{array}$ & $\begin{array}{l}\text { ccurs in the } \\
\text { bular channels } \\
\text { ns, rough (or } \\
\text { nes attached). }\end{array}$ \\
\hline GO:0005789 & $39 \begin{array}{l}\text { endoplasmic } \\
\text { reticulum memb }\end{array}$ & ibrane & cellular_component $\mathrm{T}$ & The lipid bilay & yer surrou & Inding th & e endoplasmic & reticulum. & & & & \\
\hline [KEGG Path & thway informatio & & & & & & & & & & & \\
\hline Entry & Name & & & & Descri & ption & Class & & & & & \\
\hline bta00561 & Glycerolipid met & etabolis & sm - Bos taurus (cow) & & NA & & Metabolism; & Lipid Met & etabolism & & & \\
\hline bta00830 & Retinol metaboli & lism - $\mathrm{F}$ & Bos taurus (cow) & & NA & & Metabolism; & Metabolis: & $\mathrm{sm}$ of $\mathrm{Cof}$ & factors a & and Vitamins & \\
\hline bta01100 & Metabolic pathv & iways - & - Bos taurus (cow) & & NA & & NA & & & & & \\
\hline [HCE inform & mation] & & & & & & & & & & & \\
\hline No informatio & tion. The $\mathrm{SNP}$ is $\mathrm{n}$ & not in a & a HCE. & & & & & & & & & \\
\hline [OMIA info & ormation] & & & & & & & & & & & \\
\hline No Informatic & tion Found. & & & & & & & & & & & \\
\hline
\end{tabular}

Figure 4 An example of HTML output. The first line is coloured blue, showing the items of the query. The following subsections are coloured light green or light red alternately, including SNP information, Gene information, GeneRIFs, Uniprot Protein information, QTL information, GO information, KEGG Pathway information, HCE information and OMIA information.

\section{Application}

For illustrating practical significance of Snat in facilitating functional analyses on genes/variants in the postGWAS era for bovine, Snat was implemented to annotate 20 identified significant SNPs (Additional file 4) associated with milk yield in our recent GWAS [13]. Various genomic information data (e.g., NCBI Gene, UniProt, QTLdb, etc.) related to the SNPs of interest as well as all other SNPs within $5 k$ bp region away from these 20 SNPs were mined and integrated into a raw 


\section{$\Delta$ Batch Annotation}

Please upload a file: (Format examples)

F:|works $\mid$ Query105SigSNP.TXT 测览...

\begin{tabular}{|c|c|}
\hline \multicolumn{2}{|c|}{ Annotation ( Choose any items below to integrate corresponding annotated data) } \\
\hline NCBI Gene & $\nabla$ Gene Id $\nabla$ Symbol $\nabla$ Name $\nabla$ Location (e.g. 18q11) $\nabla$ Type $\nabla$ GeneRIFs \\
\hline UniProt Protein & $\begin{array}{l}\nabla \text { Accession } \nabla \text { Recommended name } \nabla \text { Length (AA) } \nabla \text { Status (Reviewed or Unreviewed) } \\
\nabla \text { Function } \nabla \text { Subunit structure } \nabla \text { Subcellular location } \nabla \text { Post-translational modification }\end{array}$ \\
\hline QTLdb QTL & $\begin{array}{l}\nabla \text { QTL Id } \nabla \text { Trait } \nabla \text { Region (Chr,Start,Stop) } \nabla \text { Type (e.g. Significant) } \\
\nabla \text { P-value } \nabla \text { F-value } \nabla \text { Variance } \nabla \text { PubMed Id (Reference) }\end{array}$ \\
\hline Other infomation & $\begin{array}{l}\nabla \text { Gene Ontology } \nabla \text { KEGG pathway } \nabla \text { Highly conserved elements from UCSC Genome Browser } \\
\nabla \text { Online Mendelian Inheritance in Animals }\end{array}$ \\
\hline
\end{tabular}

Output
\begin{tabular}{|l|l|}
\hline $\begin{array}{l}\text { If the SNP location you input } \\
\text { refers to none of SNP in dbSNP, } \\
\text { you will find: }\end{array}$ & $\begin{array}{l}\text { The nearest SNP } \\
\text { SNPs within } 10 \mathrm{k} \text { bp region from the locus } 5 \mathrm{k} \text { bp region from the locus } \\
\text { O The nearest SNP harbored in a gene }\end{array}$ \\
\hline
\end{tabular}

B

Batch Annotation

Please upload a file: (Format examples)

F:|works|Query105SigSNP.TXT

汶览...

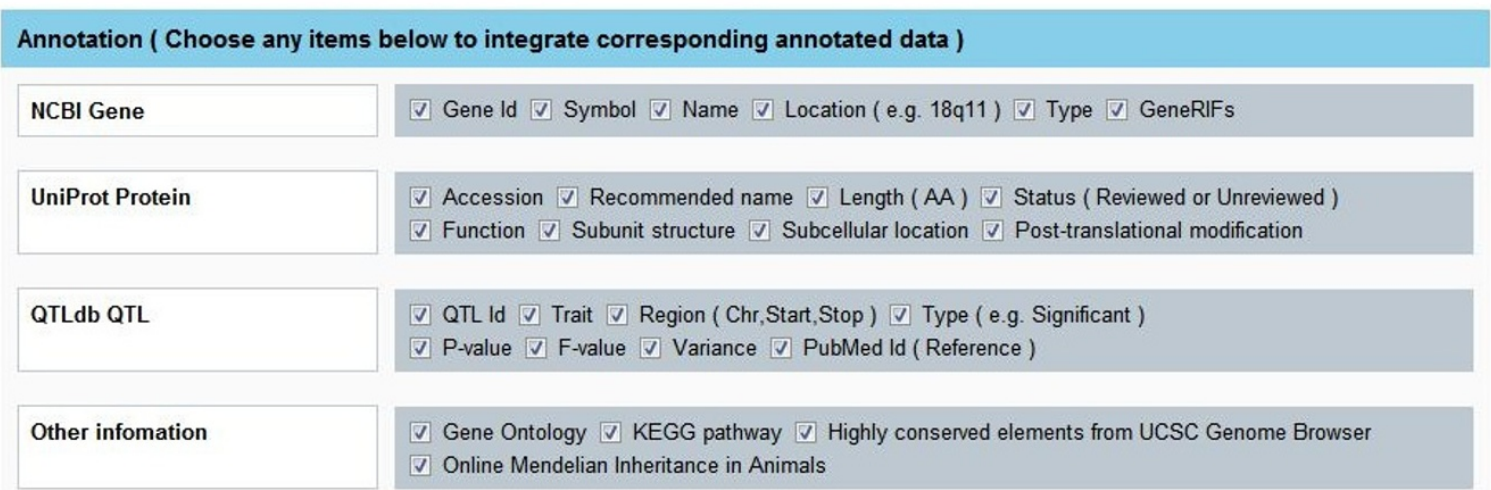

\begin{tabular}{l} 
Output \\
\begin{tabular}{l|} 
If the SNP location you input \\
refers to none of SNP in dbSNP, \\
you will find:
\end{tabular} \\
\hline
\end{tabular}

The nearest SNP 0 SNPs within $5 \mathrm{k}$ bp region from the locus

SNPs within $10 \mathrm{k}$ bp region from the locus SNPs within $30 \mathrm{k}$ bp region from the locus

The nearest SNP harbored in a gene

Figure 5 Screen shots of selected options. (A) Annotation options are all selected and the option "The nearest SNP" is specified. (B) Annotation options are all selected and the option "SNPs within 5k bp region from the locus" is specified.

text (Additional file 5). The raw annotation results were then processed by the Perl program raw2TSV.pl to generate a summary report (see Additional files 6,7) and five subdata files (see Additional files 8,9,10,11,12). From
Additional file 7, it can be found that within the $5 \mathrm{~K}$ bp region from the loci Chr14,76703 and Chr14,443937, a number of novel SNPs (see Additional file 8) are present. Among these SNPs several are either missense 
mutations or harbored in UTR. Moreover, kinds of functional information about genes around the significant SNPs from UniProt, GO and KEGG Pathway further demonstrate that these statistically significant SNPs also have potentially functional significance. For example, with respect to the locus "Chr14,443937", the annotation data of corresponding protein Q8MK44 show "May be involved in VLDL (very low density lipoprotein) CC assembly" (see Additional file 9), and the information of the corresponding pathway bta00561 shows "Glycerolipid metabolism" (see Additional file 11). All the functional information suggests that the identified SNP "Chr14,443937" likely affects milk production traits and merits follow-up functional validation study. In addition, four QTLs (Additional file 12) related to the significant SNP are associated with milk yield, further showing consistent findings with previous QTL mapping studies.

So far, several dense SNP panels, including the Affymetrix Bovine $10 \mathrm{~K}$ chip array and the Illumina $50 \mathrm{~K}$ chip array, have been increasingly implemented to identify causal mutations for economic importance in bovine under the framework of GWAS [13,14]. However, biological information related to these SNPs has not yet been provided by the chip manufacturers. It is quite daunting for researchers to conduct SNP annotation via querying various public resources manually SNP by SNP. To aid functional analyses and accurate evaluation on genes related to SNPs in GWAS, Snat was implemented herein to create SNP annotation files containing all SNPs corresponding to two commercial SNP chips. The biological information on each of these SNPs, including SNP information from dbSNP, gene information from Entrez Gene, protein features from UniProt, gene function annotations from GO, KEGG PATHWAY and OMIA, linkage information from AnimalQTLdb and conserved elements from UCSC Genome Browser Database, are integrated in these output files, which are publicly available for downloading from Snat site.

\section{Running speed}

To explore the practical feasibility of Snat, several SNP queries under different scenarios are performed for testing running speed of online annotation.

For a single SNP annotation, Snat generates outputs immediately once submitting the task. For batch annotation, 105 SNPs (Additional file 13) associated with milk production traits in our earlier GWAS [13] are adopted as the input dataset. With all query options selected as well as the option "The nearest SNP" specified (Figure $5 \mathrm{~A}$ ), it takes about 40 seconds to finish the annotation. When the output option "SNPs within $5 \mathrm{k}$ bp region from the locus" specified (Figure 5B), it takes about five minutes to achieve batch annotation of 105 SNPs. It is notable that longer time consumed for the option "SNPs within $5 \mathrm{k}$ bp region from the locus" is due to much more SNPs annotated in such situation.

Based on the above tests, the speed of online annotation is practically acceptable as running Snat.

\section{Conclusions}

We developed a SNP annotation tool which can provide comprehensive biological information related to arbitrary single nucleotide position across bovine genome. To our best knowledge, this is one of first tools focusing on SNP annotation for livestock except those for humans. Due to incorporating various sources of existing public data into a retrieved database, Snat provides an efficient and concise way for researchers to obtain annotated data of SNPs automatically via merely one query, with seldom need of further re-querying the original public databases manually.

As known to us, traditional GWAS focus on identification of statistical significance of SNPs with limited understanding of functional aspects of SNPs concerning complex traits. It is a pressing need to make an essential bridge between the outcomes from GWAS and the potential information on the function of genes and pathways. Snat offers an opportunity to achieve this goal. By Snat, researchers can sift out those SNPs indentified in GWAS with functional significance of the complex trait of interest in bovine.

We conclude that Snat can act as a complementary tool for aiding further functional analyses on genes/variants and facilitating follow-up replication studies in the post-GWAS era for bovine.

\section{Availability and requirements \\ Project name: Snat \\ Project home page: http://animalgenetics.cau.edu.cn/ snat/}

Operating system(s): Any operating system supporting Perl and MySQL

Programming language: Perl, SQL

Other requirements: Perl DBI and DBD-mysql module

License: Free for non-commercial usage

\section{Additional material}

\footnotetext{
Additional file 1: 411 SNPs involved in Illumina Bovine 50K SNP chip but not included in dbSNP. In Illumina Bovine 50K SNP chip, 52,255 markers are explicitly located in chromosomes based on Btau4.0. However, 411 out of these 52255 SNP markers are not included in dbSNP via position comparison based on Btau4.0. All these 411 SNPs are listed in the table. These SNPS can be annotated through "Any locus" function by Snat.
} 
Additional file 2: Two examples given to compare Snat with FunctSNP. The examples show the differences between the two tools on features of input data and annotation process.

Additional file 3: Detailed descriptions on annotation results by Snat. An example is provided to give a detailed explanation on the annotation reports.

Additional file 4: Information of 20 significant SNPs identified associated with milk yield. This table lists positions of 20 significant SNPs based on Btau4.0 identified associated with milk yield from our recent GWAS results (Jiang et al., 2010).

Additional file 5: The raw annotation outputs for the $\mathbf{2 0}$ significant SNPs using Snat. SNPs within $5 \mathrm{k}$ bp region away from these 20 SNPs are annotated by Snat. All the data are printed into the raw text file.

Additional file 6: A summary of annotation data for the $\mathbf{2 0}$ significant SNPs structured in TSV format. The raw annotation outputs of the 20 significant SNPs are further processed to generate a well-structured summary file in TSV format by the program raw2TSV.pl.

Additional file 7: Annotation results of the 20 significant SNPs saved in a worksheet. The summaries of annotation data for the 20 SNPS structured in TSV format are saved in the worksheet for more easily viewing.

Additional file 8: SNPs involved in dbSNP related to each of the 20 SNPs annotated. The cluster IDs (rs\#) of all SNPs harbored within $5 \mathrm{k}$ bp regions from each of these 20 SNPs are integrated. This file is extracted by the program raw2TSV.pl from the raw text file.

Additional file 9: Non-redundant UniProt protein information extracted from the raw annotation outputs for the 20 SNPs. The file is generated by the program raw2TSV.pl and contains non-redundant UniProt protein information extracted from the raw annotation outputs for the 20 SNPS.

Additional file 10: Non-redundant GO terms extracted from the raw annotation outputs for the 20 SNPs. The file is generated by the program raw2TSV.pl and contains non-redundant data of GO terms extracted from the raw annotation outputs for the 20 SNPS.

\section{Additional file 11: Non-redundant KEGG Pathway information} extracted from the raw annotation outputs for the 20 SNPs. The file is generated by the program raw2TSV.pl and contains non-redundant KEGG Pathway information extracted from the raw annotation outputs for the 20 SNPS.

Additional file 12: The numbers of QTLs and five most relevant traits corresponding to each of the 20 SNPs. The file is generated by the program raw2TSV.pl and contains the data of the numbers of QTLS and five most relevant traits corresponding to each of the 20 SNPs extracted from the raw annotation outputs.

Additional file 13: Information of 105 significant SNPs identified associated with milk production traits based on Btau4.0. This table lists positions of 105 significant SNPs based on Btau4.0 identified associated with milk production traits from our recent GWAS results (Jiang et al., 2010).

\section{List of abbreviations}

SNP: Single Nucleotide Polymorphism; CGI: Common Gate Interface; HTML: Hypertext Markup Language; TSV: Tab Separated Values; UTR: Untranslated Region

\section{Acknowledgements and Funding}

We are grateful to the anonymous reviewers for their constructive comments and suggestions that greatly improved our manuscript. This work was supported by Key Development of New Transgenic Breeds Program (2009ZX08009-156B), the National Natural Science Foundations of China (Grant No. 30972092), Beijing Municipal Natural Science Foundation (Grant No. 6102016), New-Century Training Programme Foundation for the Talents by the State Education Commission of China (NETC-10-0783), Scientific Research Foundation for the Returned Overseas Chinese Scholars, State
Education Ministry and the National High Technology Research and Development Program of China (863 Program 2011AA100302).

\section{Authors' contributions}

$J J$ and $J L$ constructed the software and drafted the manuscript. $L, B Z$ and WF participated in database construction. $J$ and $Q Z$ designed and supervised the project. All authors read and approved the final manuscript.

Received: 15 April 2011 Accepted: 7 October 2011

Published: 7 October 2011

\section{References}

1. Maglott D, Ostell J, Pruitt KD, Tatusova T: Entrez Gene: gene-centered information at NCBI. Nucleic Acids Research 2007, 35(Database):D26-D31.

2. Apweiler R, Bairoch A, Wu CH, Barker WC, Boeckmann B, Ferro S, Gasteiger E, Huang H, Lopez R, Magrane M, et al: UniProt: the Universal Protein knowledgebase. Nucleic Acids Res 2004, 32(Database):D115-119.

3. Ashburner M, Ball CA, Blake JA, Botstein D, Butler H, Cherry JM, Davis AP, Dolinski K, Dwight SS, Eppig JT, et al: Gene ontology: tool for the unification of biology. The Gene Ontology Consortium. Nat Genet 2000, 25(1):25-29.

4. Kanehisa M, Goto S, Kawashima S, Okuno Y, Hattori M: The KEGG resource for deciphering the genome. Nucleic Acids Res 2004, 32(Database): D277-280.

5. Hu ZL, Fritz ER, Reecy JM: AnimalQTLdb: a livestock QTL database tool set for positional QTL information mining and beyond. Nucleic Acids Research 2007, 35(Database):D604-D609.

6. Shen TH, Carlson CS, Tarczy-Hornoch P: SNPit: A federated data integration system for the purpose of functional SNP annotation. Computer Methods and Programs in Biomedicine 2009, 95(2):181-189.

7. Chelala C, Khan A, Lemoine NR: SNPnexus: a web database for functional annotation of newly discovered and public domain single nucleotide polymorphisms. Bioinformatics 2009, 25(5):655-661.

8. Goodswen SJ, Gondro C, Watson-Haigh NS, Kadarmideen HN: FunctSNP: an $R$ package to link SNPs to functional knowledge and dbAutoMaker: a suite of Perl scripts to build SNP databases. BMC Bioinformatics 2010, 11(1):311.

9. Sherry ST, Ward MH, Kholodov M, Baker J, Phan L, Smigielski EM, Sirotkin K: dbSNP: the NCBI database of genetic variation. Nucleic Acids Res 2001, 29(1):308-311

10. Siepel A: Evolutionarily conserved elements in vertebrate, insect, worm and yeast genomes. Genome Research 2005, 15(8):1034-1050.

11. Rhead B, Karolchik D, Kuhn RM, Hinrichs AS, Zweig AS, Fujita PA, Diekhans M, Smith KE, Rosenbloom KR, Raney BJ, et al: The UCSC Genome Browser database: update 2010. Nucleic Acids Research 2009, 38(Database):D613-D619.

12. Nicholas FW: Online Mendelian Inheritance in Animals (OMIA): a comparative knowledgebase of genetic disorders and other familial traits in non-laboratory animals. Nucleic Acids Research 2003, 31(1):275-277.

13. Jiang $L$, Liu J, Sun D, Ma P, Ding $X, Y u Y$, Zhang Q: Genome wide association studies for milk production traits in Chinese Holstein population. PLoS One 2010, 5(10):e13661.

14. Snelling WM, Allan MF, Keele JW, Kuehn LA, McDaneld T, Smith TPL, Sonstegard TS, Thallman RM, Bennett GL: Genome-wide association study of growth in crossbred beef cattle. Journal of Animal Science 2009, 88(3):837-848.

doi:10.1186/1471-2156-12-85

Cite this article as: Jiang et al:: Snat: a SNP annotation tool for bovine by integrating various sources of genomic information. BMC Genetics 2011 12:85. 